\title{
MORBILIDAD EN PACIENTES PEDIÁTRICOS SOMETIDOS A COLECISTECTOMÍA LAPAROSCÓPICA*
}

\author{
Drs. Julio Figueroa R. ${ }^{1,2,3}$, Carlos Manterola D. ${ }^{3,4,5}$, Andrés Maira S. ${ }^{2,6}$, \\ Misly Risco A. ${ }^{2,7}$, Int. Alexis Vidal A., ${ }^{2,8}$ \\ 1 Servicio de Cirugía Hospital de Puerto Montt. \\ 2 Universidad San Sebastián. \\ 3 Programa de Magíster en Ciencias, mención Cirugía, Universidad de La Frontera. Temuco. \\ ${ }^{4}$ Centro de Excelencia en estudios Morfológicos y Quirúrgicos (CEMyQ), Universidad de La Frontera. Temuco. \\ 5 Centro de Investigación en Ciencias Biomédicas, Universidad Autónoma de Chile. \\ ${ }^{6}$ Cirugía Infantil Hospital de Puerto Montt. \\ 7 Becada de Pediatría, Universidad San Sebastián. \\ 8 Interno Medicina, Universidad San Sebastián. \\ Chile.
}

\begin{abstract}
Morbidity in pediatric patients undergoing laparoscopic cholecystectomy

Introduction: Cholelithiasis is a prevalent disease in Chile. Therefore, there is sufficient evidence regarding the results of cholecystectomy in adults; however, information in pediatric population is scarce. The aim of this study is to report the results of a series of pediatric patients undergoing surgery for cholelithiasis, in terms of postoperative morbidity (POM). Material and Methods: Retrospective case series. All patients undergoing laparoscopic cholecystectomy for cholelithiasis in the period 2004 to 2013, in the Department of Surgery of Hospital de Puerto Montt, Chile, were included. The main outcome variable was POM. Other variables of interest were cause of consult, coexistence of choledocholithiasis, concomitant diseases, nature of surgery, hospital stay and mortality. Descriptive statistics were used to calculate percentages, measures of center tendency and dispersion. Results: 71 patients, with a median age of 13 years, $84.5 \%$ female, were operated. POM was $1.4 \%$ (one case of hemoperitoneum that required reoperation, Dindo-Clavien IIIb). The most frequent cause of consultation was biliary colic (95.8\% of cases). Coexistence of choledocholithiasis was determined in 6 cases $(8.5 \%)$. The most common concomitant disease was familiar microspherocytosis (4 cases, $5.6 \%)$. The surgery was elective in 62 patients $(87.3 \%)$. Median hospital stay were 2 days. The serie has no mortality. Conclusion: The observed results are comparable with those from other series of laparoscopic cholecystectomy in pediatric patients.

Key words: "Cholelithiasis"[Mesh], "Cholecystitis"[Mesh], "Cholelithiasis/surgery"[Mesh], "Cholecystectomy"[Mesh], "Cholecystectomy, Laparoscopic"[Mesh], "Choledocholithiasis"[Mesh], "Morbidity”[Mesh], "Pediatrics"[Mesh].
\end{abstract}

*Recibido el 30 de marzo de 2015 y aceptado para publicación el 29 de abril de 2015.

Los autores no refieren conflictos de interés.

Correspondencia: Dr. Carlos Manterola D. carlos.manterola@ufrontera.cl 


\section{Resumen}

Introducción: La colelitiasis es una enfermedad prevalente en Chile; por ende, existe suficiente evidencia respecto de los resultados de la colecistectomía en población adulta; sin embargo, la información en población pediátrica es escasa. El objetivo de este estudio, es reportar los resultados de una serie de pacientes pediátricos, intervenidos quirúrgicamente por colelitiasis, en términos de morbilidad postoperatoria (MPO). Material y Método: Serie de casos retrospectiva. Se incluyeron todos los pacientes pediátricos colecistectomizados vía laparoscópica en el Servicio de Cirugía del Hospital de Puerto Montt, Chile; en el período 2004 y 2013. La variable resultado fue MPO. Otras variables de interés fueron causa de consulta, coexistencia de coledocolitiasis, enfermedades concomitantes, carácter de la cirugía, estancia hospitalaria y mortalidad. Se utilizó estadística descriptiva con cálculo de porcentajes, medidas de tendencia central y dispersión. Resultados: Se intervinieron 71 pacientes, con una mediana de edad de 13 años; 84,5\% de sexo femenino. La MPO fue de 1,4\% (un caso de hemoperitoneo que requirió reoperación; Dindo-Clavien IIIb). La causa de consulta más frecuente fue el cólico biliar ( $95,8 \%$ de los casos). Se determinó coexistencia de coledocolitiasis en 6 casos $(8,5 \%)$. La enfermedad concomitante más frecuente fue microesferocitosis familiar (4 casos; 5,6\%). La cirugía fue electiva en 62 pacientes $(87,3 \%)$. La mediana de la estancia hospitalaria fue 2 días y no se reporta mortalidad. Conclusión: Los resultados observados son comparables con los de otras series de colecistectomía laparoscópica en pacientes pediátricos.

Palabras clave: Colelitiasis, colecistitis, colecistectomía, colecistectomía laparoscópica, coledocolitiasis, morbilidad postoperatoria, cirugía pediátrica.

\section{Introducción}

La colelitiasis es una entidad prevalente en Chile (prevalencia superior al 50\% en mujeres) ${ }^{1}$. Sin embargo, no existen cifras precisas de prevalencia e incidencia en población pediátrica ${ }^{2-6}$. Por otra parte, el cólico biliar, es una causa poco frecuente de dolor abdominal y de consulta en niños ${ }^{2-7}$.

En nuestro medio, la colelitiasis en pacientes pediátricos, es resuelta de forma frecuente por cirujanos de adultos.

No existen estudios que caractericen a la población chilena menor de 15 años con colelitiasis desde el punto de vista de factores de riesgo, como tampoco respecto de la morbilidad postoperatoria (MPO); razón por la cual, nos pareció pertinente reportar los resultados de nuestra experiencia en este tema.

Este manuscrito ha sido redactado siguiendo la lista de chequeo para estudios observacionales descriptivos $^{8}$.

El objetivo general de este estudio, es reportar los resultados de una serie de pacientes pediátricos, intervenidos quirúrgicamente por colelitiasis, en términos de MPO. Los objetivos específicos son: Determinar la frecuencia de patología asociada; y de coledocolitiasis detectada en el estudio peroperatorio.

\section{Material y Método}

\section{Tipo de estudio}

Serie de casos retrospectiva.

\section{Población}

Se incluyeron pacientes menores de 15 años intervenidos quirúrgicamente por colelitiasis, por cirujanos de adultos, en el Servicio de Cirugía del hospital de Puerto Montt, Chile, en el período de enero de 2004 a mayo de 2013. No se aplican criterios de exclusión.

\section{Protocolo de estudio}

Se confeccionó una hoja de recogida de datos en las que se registraron las características biodemográficas y clínicas de los pacientes, las hojas de descripción operatoria; y evolución postoperatoria hasta su alta hospitalaria.

\section{Variables de interés}

La variable resultado principal fue desarrollo de MPO (la que se trabajó de forma dicotómica [no/sí], y por gravedad, aplicando la escala de Dindo y Clavien ${ }^{9}$; para lo que se exigió un seguimiento mínimo de 30 días). Otras variables de interés fueron causa de consulta, coexistencia de coledocolitiasis peroperatoria diagnosticada con imágenes, enfermedades concomitantes, carácter de la cirugía (urgencia o electiva); tiempo quirúrgico, necesidad de reintervenciones, estancia hospitalaria (días) y mortalidad.

\section{Protocolo de seguimiento}

Se realizó control clínico al $7^{\circ}$ día (curación y retiro de puntos); y luego a los 15 y 30 días del postoperatorio. 


\section{Tratamiento realizado}

En la totalidad de los casos, se realizó colecistectomía laparoscópica con técnica americana.

\section{Sesgos}

Para reducir el sesgo de medición, un investigador independiente, distinto de los cirujanos que operaron; recolectó los datos.

\section{Plan de análisis}

Se realizó análisis exploratorio de los datos. Se utilizó estadística descriptiva con cálculo de porcentajes, medidas de tendencia central y dispersión.

\section{Aspectos éticos}

Se observaron las guías éticas para investigación en seres humanos (Declaración de Helsinki) y los datos se trataron confidencialmente.

\section{Fuentes de financiación}

El estudio no contó con fuentes formales de financiamiento.

\section{Resultados}

En el período estudiado, se intervino un total de 71 pacientes, con una edad promedio de $12,5 \pm 2,0$ años y mediana de edad de 13 años; $84,5 \%$ de los cuales eran de sexo femenino. Las variables clínicas se describen en las Tablas 1 y 2.

La causa de consulta más frecuente fue el cólico biliar (95,8\% de los pacientes), el resto de las manifestaciones clínicas se detalla en la Tabla 3.

La cirugía fue electiva o programada en el $87,3 \%$ de los casos $(\mathrm{n}=62)$. Se determinó coexistencia de coledocolitiasis en 6 casos $(8,5 \%)$.

La enfermedad concomitante más frecuente fue microesferocitosis familiar, con 4 casos $(5,6 \%)$. El resto de éstas se detalla en la Tabla 4.

El tiempo quirúrgico fue de $51 \pm 13$ min y la mediana de la estancia hospitalaria fue 2 días.

Con un seguimiento de al menos un mes, la MPO fue de $1,4 \%$. Se trata de un caso de hemoperitoneo que requirió cirugía de urgencia post colecistectomía (IIIb de Dindo y Clavien).

La serie no reporta mortalidad.

\section{Discusión}

La colelitiasis en menores de 15 años, y a diferencia de los adultos, es una causa de consulta poco frecuente tanto de electiva como de urgencia; sin embargo, parece estar aumentando con el mayor acceso a estudio con imágenes y a la calidad de estos métodos diagnósticos ${ }^{10}$.
Tabla 1. Distribución de variables continuas preoperatorias de los pacientes en estudio $(n=71)$

\begin{tabular}{|lccc|}
\hline Variable & Promedio \pm DE & Mediana & $\begin{array}{c}\text { Valores } \\
\text { extremos }\end{array}$ \\
\hline $\begin{array}{l}\text { Edad (años) } \\
\begin{array}{l}\text { Tiempo } \\
\text { quirúrgico (min) }\end{array}\end{array}$ & $12,5 \pm 2,0$ & 13 & $6-15$ \\
$\begin{array}{l}\text { Estancia } \\
\text { hospitalaria (días) }\end{array}$ & $1,8 \pm 0,7$ & 2 & $13-60$ \\
$\begin{array}{l}\text { Seguimiento } \\
\text { (meses) }\end{array}$ & $1,1 \pm 0,1$ & 1 & $1-6$ \\
\hline
\end{tabular}

Tabla 2. Distribución de variables categóricas en los pacientes en estudio $(n=71)$

\begin{tabular}{|lcr|}
\hline Variable & n casos & \% \\
Sexo & & \\
$\quad$ Femenino & 60 & 84,5 \\
$\quad$ Masculino & 11 & 15,5 \\
Antecedentes de pancreatitis & & \\
$\quad$ Sí & 11 & 15,5 \\
$\quad$ No & 60 & 84,5 \\
Necesidad de realizar CRE* & & \\
Sí & 7 & 9,9 \\
No & 64 & 90,1 \\
\hline
\end{tabular}

*CRE: Colangiografía retrógrada endoscópica.

Tabla 3. Manifestaciones clínicas verificadas en los pacientes en estudio $(n=71)$

\begin{tabular}{|lcc|}
\hline Síntomas/Signos & n casos & \% \\
\hline Cólico biliar & 68 & 95,8 \\
Vómitos & 38 & 53,5 \\
Ictericia & 11 & 15,5 \\
Fiebre & 6 & 8,5 \\
\hline
\end{tabular}

Los pacientes presentaron más de un síntoma y signo.

Tabla 4. Enfermedades concomitantes verificadas en los pacientes en estudio $(n=71)$

\begin{tabular}{|lcr|}
\hline Enfermedades concomitantes & n casos & \% \\
\hline Ninguna & 61 & 85,9 \\
Microesferocitosis familiar & 4 & 5,6 \\
Anemia hemolítica & 2 & 2,8 \\
Síndrome de Down & 2 & 2,8 \\
Linfoma de Hodgkin & 1 & 1,4 \\
Resistencia a la insulina & 1 & 1,4 \\
Total & 71 & 100,0 \\
\hline
\end{tabular}


En nuestro país el número de publicaciones sobre el tema es escaso, tal vez debido a que es poco frecuente. A partir de 1976, sólo hemos podido encontrar 3 artículos ${ }^{2,3,11}$. Existen otros tres reportes publicados anteriormente, entre 1930 y 1955.

Si comparamos la distribución por sexo vemos que al igual que en los adultos el predominio es femenino. En esta serie, un $84 \%$ son niñas; cifra superior a la reportada en otras series ${ }^{4-6,12-15}$.

La coexistencia de coledocolitiasis $(8,5 \%$ de los casos), es comparable con otras experiencias, tanto pediátricas como de adultos, por lo que no parece haber diferencia en la presentación de esta comorbilidad en este grupo de edad ${ }^{13,16}$.

La morbimortalidad registrada, es similar a series de adultos, contrario a lo que uno pudiese pensar por ser sujetos anatómicamente más pequeños, hecho que pudiera significar un mayor riesgo de $\mathrm{MPO}^{4,17}$.

La estadía hospitalaria (mediana de 2 días), es mayor tanto a lo que se observa en la práctica cotidiana como en reportes de colecistectomía laparoscópica en población adulta; lo que podría explicarse dado el temor de un "alta precoz" en pacientes, que además de ser pediátricos, son principalmente de domicilio rural (el hospital de Puerto Montt, es centro de referencia de toda la Región de Los lagos). De todos modos, en comparación con otras series pediátricas la estancia hospitalaria es similar ${ }^{17-20}$.

A modo de conclusión, podemos señalar que colelitiasis en menores de 15 años no es muy habitual pero su tratamiento mediante una colecistectomía laparoscópica presenta una frecuencia de MPO que es baja, similar a la cirugía en adultos y con otras series de pacientes pediátricos encontradas en la literatura ${ }^{16,17}$.

\section{Referencias}

1. Covarrubias C, del Pino G, Ferreiro O, Nervi F. Epidemiology and natural history of biliary calculi. Implication for clinical management. Rev Med Chile 1992;120:432-8.

2. Harris P, Chateau B, Miquel JF, Zavala A, Montes P, Herrera JM, et al. Cholelithiasis in children: a clinical and morphological study. Rev Med Chile 2003;131:3745.

3. Cerda J, Torres F, Aguirre J, Aldunate G, David W, Vildósola C. Colecistitis y colelitiasis en niños. Rev Chil Pediatr. 1976;47:133-7.

4. Wesdorp I, Bosman D, de Graaff A, Aronson D, van der Blij F, Taminiau J. Clinical presentations and predisposing factors of cholelithiasis and sludge in children. $\mathrm{J}$ Pediatr Gastroenterol Nutr. 2000;31:411-7.
5. Cozcolluela Cabrejas MR, Sanz Salanova LA, MartínezBerganza Asensio MT, Gómez Herrero H, Mellado Santos JM, Miranda Orella L, et al. Childhood cholelithiasis in a district hospital. An Pediatr (Barc). 2007;66:611-4.

6. Ruibal Francisco J, Aleo Luján E, Álvarez Mingote A, Piñero Martínez E, Gómez Casares R. Childhood cholelithiasis. Analysis of 24 patients diagnosed in our department and review of 123 cases published in Spain. An Esp Pediatr. 2001; 54:120-5.

7. Mehmood A, Khan MA. Biliary stones: an atypical cause of abdominal pain in paediatric age group. J Pak Med Assoc. 2010;60:1042-4.

8. Manterola C, Astudillo P, Grupo MINCIR. Checklist for Reporting of Descriptive Observational Studies. MINCIR Initiative. Int J Morphol 2013;31:115-120.

9. Clavien PA, Barkun J, de Oliveira ML, Vauthey JN, Dindo D, Schulick RD, et al. The Clavien-Dindo classification of surgical complications: five-year experience. Ann Surg. 2009;250:187-96.

10. Waldhausen JH, Benjamin DR. Cholecystectomy is becoming an increasingly common operation in children. Am J Surg. 1999;177:364-7.

11. Matus C, Romanini MV, Correía G, Ibáñez R, Velozo L, Romero P. Colecistitis en niños. Pediatría (Chile) 2001;44:19-21.

12. Kelley-Quon LI, Dokey A, Jen HC, Shew SB. Complications of pediatric cholecystectomy: impact from hospital experience and use of cholangiography. J Am Coll Surg. 2014;218:73-81.

13. Zeidan MM, Pandian TK, Ibrahim KA, Moir CR, Ishitani MB, Zarroug AE. Laparoscopic cholecystectomy in the pediatric population: a single-center experience. Surg Laparosc Endosc Percutan Tech. 2014;24:248-50

14. Mehta S, López ME, Chumpitazi BP, Mazziotti MV, Brandt ML, Fishman DS. Clinical characteristics and risk factors for symptomatic pediatric gallbladder disease. Pediatrics 2012;129:e82-8.

15. Kumar R, Nguyen K, Shun A. Gallstones and common bile duct calculi in infancy and childhood. Aust $\mathrm{N} \mathrm{Z} \mathrm{J}$ Surg. 2000;70:188-91.

16. Bonnard A, Seguier-Lipszyc E, Liguory C, Benkerrou M, Garel C, Malbezin S, et al. Laparoscopic approach as primary treatment of common bile duct stones in children. J Pediatr Surg. 2005;40:1459-63.

17. Esposito C, González Sabin MA, Corcione F, Sacco R, Esposito G, Settimi A. Results and complications of laparoscopic cholecystectomy in childhood. Surg Endosc. 2001;15:890-2.

18. Ainsworth AP, Axelsen AR, Rasmussen L. Cholecystectomy in children. Dan Med Bull. 2010;57:A4201.

19. Agarwal P, Bagdi RK, Balagopal S, Madhu R, Balamourougane P. Pediatric Gowda DJ, et al. Laparoscopic cholecystectomy for cholelithiasis in children. J Indian Assoc Pediatr Surg. 2009;14:204-6. 